\title{
A Two Dimensional Lagrangian Model with Extended Supersymmetry and Infinitely Many Constants of Motion
}

\author{
E. Napolitano and S. Sciuto
}

Istituto di Fisica Teorica dell 'Università di Torino, Sezione di Torino dell' Istituto Nazionale Fisica Nucleare, I-10125 Torino, Italy

\begin{abstract}
The reduction of the supersymmetric graded $\frac{S U(2 \mid 1)}{S\left(U_{2} \times U_{1}\right)} \sigma$-model is discussed. If no extra constraint is imposed, one gets a set of two coupled equations (involving two scalar superfields) which generalizes the supersymmetric sine-Gordon equation. It is shown that these equations, which can be derived by a supersymmetric Lagrangian, reproduce in the bosonic limit the reduced version of the $O(4) \sigma$-model (Pohlmeyer, Lund Regge, Getmanov model). Moreover the associate linear set and an infinite set of local conservation laws for this new supersymmetric model are exhibited. It turns out that, beyond the spinorial charge which generates the supersymmetry transformations, another unexpected spinorial charge is conserved; then the model appears to be invariant under $N=2$ extended supersymmetry.
\end{abstract}

\section{Introduction}

A supersymmetric generalization of the sine-Gordon equation was proposed some years ago by Di Vecchia, Ferrara and Witten [1]. Later an infinite set of local conservation laws were found for this model [2]; it was shown that they survive also at the quantum level [3] and the $S$-matrix was calculated [4]. The proof of the classical conservation laws was given $[5,6]$ by introducing a "Lax set" of linear equations associated to the super sine-Gordon equation.

An interesting (purely bosonic) generalization of the sine-Gordon equation was independently proposed by Pohlmeyer, Lund and Regge [7] who showed its relationship with the $O(4) \sigma$-model, and by Getmanov [8]; this model was named the Complex sine-Gordon by Getmanov, but we will call it the GLRP model in order to avoid confusion with a different complexification of the sine-Gordon equation (Complex sine-Gordon II) which was found later $[9,10]$. It is very easy to write a supersymmetric generalization of the GLRP model; however for the most obvious supersymmetrization no associate linear set has been found (actually the 
attempts to find a supersymmetric extension of the linear set associated to the GLRP led to the discovery of a new model, whose bosonic limit is the Complex sineGordon model II [10]).

In [10] and [11] a general scheme to build supersymmetric models endowed with an associate linear set was proposed; it was then possible to write [11] the linear set associated to a new supersymmetric model related to the GLRP model. This model involves 3 coupled scalar superfields, and in the purely bosonic limit gives two sets of decoupled equations: one set reproduces the 2 coupled equations of the GLRP model, the other one is simply the sine-Gordon equation. The sine-Gordon field and the GLRP fields interact through their fermionic counterparts and then the supersymmetric equations cannot be decoupled.

By looking at the GLRP model as a special form of the principal chiral $S U$ (2) model [12], in the present paper we succeed to give a new supersymmetric version, which does not involve any extra field and which is endowed of an associate linear set and of an infinite set of local conservation laws. Interestingly enough, the model shows a larger supersymmetry than the one built in at the beginning: the model is described by superfields of simple supersymmetry, but two spinorial charges appear to be conserved, generating then an extended supersymmetry.

In Sect. 2 we present the Lagrangian and the equations of motion of this model in terms of superfields. In Sects. 3 and 4 we explain how the equations of motion were obtained: after reviewing (Sect. 3) the general method of refs. [10] and [11], in Sect. 4 we apply it to the reduction of the supersymmetric graded $S U(2 \mid 1)$ $\sigma$-model and we get the linear set associate to our model. In Sect. 5 we write the recursive formula for the infinite set of local conservation laws and briefly discuss the new unexpected supersymmetry. Finally in the Appendix the Lagrangian and the equations of motion are written in terms of ordinary fields.

\section{The Lagrangian and the Equations of Motion}

The model we wish to discuss is described by the following action ${ }^{1}$.

$$
S=i \int d^{2} x d^{2} \theta\left\{-\frac{i}{4}\left(D^{\alpha} \Phi D_{\alpha} \Phi+\cot ^{2} \Phi D^{\alpha} H D_{\alpha} H\right)-V(\Phi, H)\right\}
$$

where

$$
V(\Phi, H)=m \cos \Phi \cos H,
$$

and $\Phi$ and $H$ stand for real superfields:

$$
\begin{aligned}
& \Phi(x, \theta)=\varphi(x)+i \theta^{\alpha} \psi_{\alpha}(x)+\frac{i}{2} \theta^{\alpha} \theta_{\alpha} F(x), \\
& H(x, \theta)=h(x)+i \theta^{\alpha} \chi_{\alpha}(x)+\frac{i}{2} \theta^{\alpha} \theta_{\alpha} G(x) .
\end{aligned}
$$

The corresponding equations of motion are:

1 We use the notation $d \theta$ inside the Berezin integrals [13] in order to avoid any confusion with the differentials $d \theta$ which will come into play in the next sections 


$$
\begin{aligned}
\frac{i}{2} D^{\alpha} D_{\alpha} \Phi+\frac{i}{2} \frac{\cos \Phi}{\sin ^{3} \Phi} D^{\alpha} H D_{\alpha} H+m \sin \Phi \cos H & =0, \\
\frac{i}{2} D^{\alpha} D_{\alpha} H-\frac{i}{\sin \Phi \cos \Phi} D^{\alpha} \Phi D_{\alpha} H+m \frac{\sin ^{2} \Phi}{\cos \Phi} \sin H & =0 .
\end{aligned}
$$

Of course, for $H=0$, the set (2.4) collapses into the super sine-Gordon equation $D_{1} D_{2} \Phi=i m \sin \Phi$.

We postpone to the Appendix the expression of the Lagrangian and of the field equations in terms of component fields. Here we only discuss the bosonic limit obtained by sending to zero the fermionic fields $\psi_{\alpha}$ and $\chi_{\alpha}$ in Eqs. (2.3), in order to exhibit the rôle of the auxiliary fields $F$ and $G$.

By substituting into the action (2.1) the superfields (2.3) with $\psi=\chi=0$, and after integration on the $\theta$ variables, one gets:

$$
\begin{aligned}
S_{b}= & \int d^{2} x\left\{-\frac{1}{2}\left[\partial^{\mu} \varphi \partial_{\mu} \varphi-F^{2}+\cot ^{2} \varphi\left(\partial^{\mu} h \partial_{\mu} h-G^{2}\right)\right]\right. \\
& +m(F \sin \varphi \cos h+G \cos \varphi \sin h)\} .
\end{aligned}
$$

The corresponding equations of motion now read:

$$
\begin{gathered}
F=-m \sin \varphi \cos h, \\
G=-m \frac{\sin ^{2} \varphi}{\cos \varphi} \sin h, \\
\square \varphi+\frac{\cos \varphi}{\sin ^{3} \varphi}\left(\partial^{\mu} h \partial_{\mu} h-G^{2}\right)+m(F \cos \varphi \cos h-G \sin \varphi \sin h)=0, \\
\square h-\frac{4}{\sin ^{2} \varphi} \partial^{\mu} \varphi \partial_{\mu} h+m \tan ^{2} \varphi(G \cos \varphi \cos h-F \sin \varphi \sin h)=0 .
\end{gathered}
$$

By inserting Eqs. (2.6) into Eqs. (2.7) and (2.5) one finally gets the equations for the propagating fields $\varphi$ and $h$ :

$$
\begin{array}{r}
\square \varphi+\frac{\cos \varphi}{\sin ^{3} \varphi} \partial^{\mu} h \partial_{\mu} h-m^{2} \sin \varphi \cos \varphi=0, \\
\square h-\frac{4}{\sin 2 \varphi} \partial^{\mu} \varphi \partial_{\mu} h=0,
\end{array}
$$

and the corresponding action

$$
S_{b}=-\frac{1}{2} \int d^{2} x\left(\partial^{\mu} \varphi \partial_{\mu} \varphi+\cot ^{2} \varphi \partial^{\mu} h \partial_{\mu} h+m^{2} \sin ^{2} \varphi\right) .
$$

One easily realizes that Eqs. (2.8) reproduce the equations of motion of the Getmanov, Pohlmeyer, Lund-Regge model [7,8]. It is worthwhile to observe that the supersymmetric generalization of this model described by Eqs. (2.1) and (2.4) is not unique at all: for instance, the insertion of the potential $V(\Phi)=m \cos \Phi$ (instead of (2.2)) into the action (2.1), would lead to the same bosonic limit. However the supersymmetrization (2.1) of GLRP has the advantage that its equations of 
motion can be interpreted as integrability conditions of a linear set, as we shall prove in Sect. 4. As a consequence, the model admits an infinite set of local conservation laws.

Moreover, we recall that a different model, still endowed with an associate linear set, can be derived from the action (2.1) with the potential $V(\Phi)=m \frac{\cos 2 \Phi}{2}$. But, as shown in ref. [10] where this model is introduced, the presence of $2 \Phi$ instead of $\Phi$ is enough to radically change the underlying group theoretical structure. Its bosonic limit no longer gives the GLRP equations, but another model (complex sine-Gordon II, $[9,10]$ ) associated with the $S U(3) / O(3)$ symmetric space $[11,14]$.

\section{Exterior Calculus on Superspace and Associate Linear Set}

In this section we briefly review the formalism outlined in refs. $[10,11]$ in order to get the linear set associated to the equations of motion (2.4).

A linear set of first order differential equations can be elegantly written by means of the formalism of differential forms

$$
d V=\omega V,
$$

where $V$ is a column of 0 -forms, $\omega$ is a matrix of 1 -forms and $d$ means exterior derivative.

The integrability condition of the linear set (3.1) is

$$
R=0,
$$

where $R$ is the following 2 -form (the "curvature"):

$$
R \stackrel{\operatorname{def}}{=} d \omega-\omega \wedge \omega
$$

The Eqs. (3.1) and (3.2) can be written on any manifold irrespective of its dimensionality. Obviously two-dimensional space time has the interesting peculiarity that Eq. (3.2) reduces to a single matrix equation, as any 2-form is proportional to $d x^{0} \wedge d x^{1}$. However, in order to build two-dimensional supersymmetric models, Eqs. (3.1) and (3.2) must be written on the superspace parametrized by the ordinary variables $x^{\mu}(\mu=0,1)$ and by the Grassmann variables $\theta^{\alpha}(\alpha=1,2)$.

At first sight one has to deal with much more components of Eq. (3.2) than in the ordinary two-dimensional space; in fact a 2-form on $(2+2)$ superspace has components along 8 independent directions ${ }^{2}$ :

$$
\begin{gathered}
d \theta^{\{\alpha} \wedge d \theta^{\beta\}} \\
d \theta^{\alpha} \wedge d x^{\mu} \\
d x^{[\mu} \wedge d x^{\nu]} .
\end{gathered}
$$

2 The brackets \{\} and [ ] mean symmetrization and antisymmetrization respectively, and recall the commutation properties of the wedge product among the differentials $d \theta^{\alpha}$ and $d x^{\mu}$ 
Actually it happens that only 3 out of the 8 equations contained in Eq. (3.2) are independent; even more, a single equation gives the most relevant information [10]. To prove this property it is convenient to use the contravariant basis:

$$
\begin{aligned}
& E^{\alpha}=d \theta^{\alpha} \\
& e^{\mu}=d x^{\mu}-i d \theta^{\alpha}\left(\gamma^{\mu} \theta\right)_{\alpha}
\end{aligned}
$$

of left invariant forms under rigid supersymmetry transformations

$$
\begin{aligned}
\theta^{\alpha} & \rightarrow \theta^{\alpha}+\varepsilon^{\alpha}, \\
x^{\mu} & \rightarrow x^{\mu}-i \varepsilon^{\alpha}\left(\gamma^{\mu} \theta\right)_{\alpha} ;
\end{aligned}
$$

let us remark that the basis (3.5) is dual to the basis

$$
\begin{aligned}
& D_{\alpha}=\frac{\partial}{\partial \theta^{\alpha}}+i\left(\gamma^{\mu} \theta\right)_{\alpha} \frac{\partial}{\partial x^{\mu}} \\
& \partial_{\mu}=\frac{\partial}{\partial x^{\mu}}
\end{aligned}
$$

of tangent vectors (or supersymmetric covariant derivatives). By inserting the 2-form

$$
R=E^{\alpha} \wedge E^{\beta} R_{\alpha \beta}+E^{\alpha} \wedge e^{\mu} R_{\alpha \mu}+e^{\mu} \wedge e^{v} R_{\mu \nu}
$$

into the Bianchi identity

$$
d R=\omega \wedge R-R \wedge \omega
$$

and by using the Maurer Cartan equation for the graded translation group

$$
d e^{\mu}=i E^{\alpha}\left(\gamma^{\mu} E\right)_{\alpha}
$$

(which directly follows from the definition (3.5)) one easily gets [15] that the equation

$$
R_{\alpha \beta}=0
$$

implies the equations

$$
\begin{aligned}
& R_{\mu \alpha}=0, \\
& R_{\mu \nu}=0,
\end{aligned}
$$

In other words, in the basis (3.5), the necessary and sufficient condition that the 2-form $R$ vanish is the vanishing of its components along the directions $d \theta^{\{\alpha} \wedge d \theta^{\beta\}}$. $\Omega$ :

It is useful to write Eq. (3.11) in terms of the components $\Omega_{\alpha}, \omega_{\mu}$ of the 1 -form

$$
\omega=E^{\alpha} \Omega_{\alpha}+e^{\mu} \omega_{\mu}
$$

to this aim it is convenient to use the notations of ref. [10] and introduce the truncated 1-form ( $t$-form)

$$
\widehat{\Omega}=E^{\alpha} \Omega_{\alpha} \equiv d \theta^{\alpha} \Omega_{\alpha},
$$


and the truncated exterior derivative

$$
\hat{D}=E^{\alpha} D_{\alpha} \equiv d \theta^{\alpha} D_{\alpha} .
$$

In this language, the definitions (3.3), (3.8), (3.13) and Eq. (3.10) give:

$$
E^{\alpha} \wedge E^{\beta} R_{\alpha \beta}=i E^{\alpha}\left(\gamma^{\mu} E\right)_{\alpha} \omega_{\mu}+\hat{D} \hat{\Omega}-\hat{\Omega} \wedge \hat{\Omega}
$$

Moreover, by inserting the definition

$$
\hat{D} \hat{\Omega}-\hat{\Omega} \wedge \hat{\Omega} \stackrel{\text { def }}{=} \hat{\rho}=E^{\alpha} \wedge E^{\beta} \rho_{\alpha \beta}
$$

in Eq. (3.16), Eq. (3.11) becomes:

$$
\begin{aligned}
& \rho_{12}=0, \\
& \omega_{+}=-i \rho_{11}, \\
& \omega_{-}=i \rho_{22},
\end{aligned}
$$

where light cone coordinates

$$
x^{ \pm}=\frac{x^{1} \pm x^{0}}{2}, \omega_{ \pm}=\omega_{1} \pm \omega_{0}
$$

have been used.

Then the most convenient way to discuss the linear set (3.1) in the supersymmetric case is to start from the truncated linear set

$$
\hat{D} V=\hat{\Omega} V,
$$

whose integrability condition is the single matrix equation (3.18); following ref. [10], it can be written in the form:

$$
\hat{D} \hat{\Omega}-\hat{\Omega} \wedge \hat{\Omega} \doteq 0,
$$

where the symbol $\doteq 0$ means that the coefficient of $d \theta^{1} \wedge d \theta^{2}$ in the left hand side must vanish. The Eq. (3.18) does not involve the coefficients of $d \theta^{1} \wedge d \theta^{1}$ and of $d \theta^{2} \wedge d \theta^{2}$; however the calculation of these coefficients is very useful, because Eqs. (3.19) can be used to define $\omega_{\mu}$ in terms of $\Omega_{\alpha}$.

Then the truncated linear set (3.20) can be completed by adding the equations

$$
\partial_{\mu} V=\omega_{\mu} V
$$

with the usual consistency conditions:

$$
\partial_{\mu} \omega_{v}-\partial_{v} \omega_{\mu}-\left[\omega_{\mu}, \omega_{v}\right]=0 .
$$

Let us remark again that Eq. (3.22), which coincides with Eq. (3.12b), is not a new one but is implied by Eq. (3.18) on $\Omega_{\alpha}$ and by the definition (3.19) of $\omega_{\mu}$.

As we will see in our specific example, the $\theta$ independent part of Eq. (3.22) is very useful to give the equations of motion in terms of ordinary fields, while Eq. (3.18) gives the same equations in terms of superfields.

In order to build supersymmetric models, two possibilities are envisaged in ref. [11]: 
i) The $t 1$-form $\hat{\Omega}$ (as the complete 1 -form $\omega$ ) takes values on an algebra $\widehat{\mathfrak{F}}$ graded according to a $Z_{2}$ grading

$$
\hat{\mathfrak{G}}=\mathfrak{b}_{0} \oplus \mathfrak{G}_{1},
$$

with the (anti) commutation relations

$$
A_{a} A_{b}-(-)^{a b} A_{b} A_{a} \in \mathfrak{G}_{a+b(\bmod 2)}, A_{a} \in \mathfrak{C}_{a},
$$

and $\hat{\Omega}$ is chosen to be

$$
\hat{\Omega}=\mathfrak{a}+K,
$$

where $\mathfrak{a} \in \mathfrak{b}_{0}$ is a matrix of bosonic $t 1$-forms and $K \in \mathfrak{b}_{1}$ of fermionic ones;

ii) Again $\hat{\Omega} \in \mathfrak{G}$, but now a $Z_{4}$ grading is considered ${ }^{3}$

$$
\hat{\mathfrak{G}}=\mathfrak{G}_{0} \oplus \mathfrak{G}_{1} \oplus \mathfrak{G}_{2} \oplus \mathfrak{G}_{3},
$$

with the (anti) commutation relations:

$$
A_{a} A_{b}-(-)^{a b} A_{b} A_{a} \in \mathfrak{G}_{a+b(\bmod 4)} .
$$

The $t 1$-form $\hat{\Omega}$ is decomposed in the following way:

$$
\hat{\Omega}=\mathfrak{a}+d \theta^{1} K_{1}+d \theta^{2} K_{2},
$$

where $\mathfrak{a} \in \mathfrak{G}_{0}$ is a matrix of bosonic forms, and $K_{1} \in \mathfrak{b}_{1}, K_{2} \in \mathfrak{G}_{3}$ are matrices of bosonic superfields (hence $d \theta^{1} K_{1}$ and $d \theta^{2} K_{2}$ are fermionic $t 1$-forms) ${ }^{4}$.

Actually in [11] only the case ii) is discussed in detail, as the aim of that work is to build supersymmetric generalizations of bosonic models related to $G / H$ Riemannian symmetric spaces. ( $G$ is the group generated by the even subalgebra $\mathfrak{\mathfrak { F }}_{0} \oplus \mathfrak{\mathfrak { F }}_{2}$, and $H$ is the subgroup generated by $\mathfrak{\mathfrak { F }}_{0}$.) In particular the super

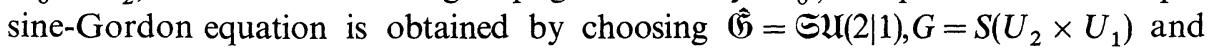
$H=S O(2)$; the relevant symmetric space in the bosonic limit is $S U(2) / S O(2)$ and the corresponding model is the sine-Gordon one. The most natural extension comes from the choice $\left(\hat{\mathfrak{5}}=\mathfrak{S U}(3 \mid 1), G=S\left(U_{3} \times U_{1}\right), H=S O(3)\right.$, and in the bosonic limit gives the complex sine-Gordon model II $[9,10]$ associated with the symmetric space $S U(3) / S O(3)[14]$.

According to the scheme ii), a supersymmetric generalization of the GLRP model can be found by exploiting its relationship with the symmetric space

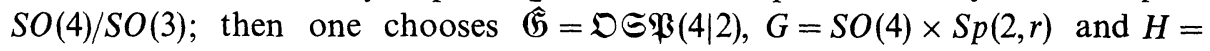
$S O(3) \times S O(2)$. The bosonic limit of this supersymmetric model [11] is the "direct sum" of two decoupled sets of equations: one (the GLRP model) is related to the symmetric space $S O(4) / S O(3)$, the other (the sine-Gordon model) is related to the symmetric space $\operatorname{Sp}(2, r) S O(2)$.

On the contrary in the present paper we will show that the case i), with the simplest nontrivial choice $\tilde{\mathfrak{G}}=\mathfrak{S} \mathfrak{U}(2 \mid 1), \mathfrak{F}_{0}=\mathfrak{S}\left(\mathfrak{H}_{2} \oplus \mathfrak{U}_{1}\right)$, reproduces the supersymmetric model described by the action (2.1).

3 We thank J. Lukierski for a useful comment on this point (see also [16])

4 Note the $\hat{\Omega}$ has vanishing projection on $\mathfrak{G}_{2}$; however one can see, by Eqs. (3.19) and (3.27) that $\omega$ does have non-vanishing components along $\mathfrak{G}_{2}$ 


\section{The Reduced $S U(2 \mid 1) / S\left(U_{2}{ }^{*} U_{1}\right)$ Model}

In order to build the linear set associated to the model proposed in Sect. 2 we will apply the formalism of Sect. 3 to the supersymmetric graded $S U(2 \mid 1) / S\left(U_{2} \times U_{1}\right)$ $\sigma$-model [17]. Sending the reader to ref. [17] for a more detailed treatment of supersymmetric graded $\sigma$-models, in the present paper we write our model already in the form most suitable for the reduction.

In the linear set (3.20), with

$$
V-\left(\begin{array}{l}
V_{1} \\
V_{2} \\
V_{3}
\end{array}\right)\left(V_{2}, V_{3} \text { are bosonic superfields and } V_{1} \text { is a fermionic one }\right)
$$

the $t 1$-form $\hat{\Omega}$ is chosen to take values in the fundamental representation of the graded Lie algebra $\Xi \mathfrak{U}(2 \mid 1)$ :

$$
\begin{gathered}
\hat{\Omega}=-g^{-1} \hat{D} g=\left(\begin{array}{cc}
\hat{a} & -\hat{k}^{\dagger} \\
\hat{k} & \hat{A}
\end{array}\right), g \in S U(2 \mid 1), \\
(\operatorname{Str} \hat{\Omega} \equiv \hat{a}-\operatorname{Tr} \hat{A}=0),
\end{gathered}
$$

where $\hat{A} \in \mathfrak{U}(2)$ is a $2 \times 2$ antihermitian matrix of bosonic $t 1$-forms

$$
\hat{A}=d \theta^{1} A_{1}+d \theta^{2} A_{2},
$$

( $A_{1}$ and $A_{2}$ are matrices of fermionic superfields), and $\hat{k}$ is a column of fermionic $t 1$-forms

$$
\hat{k}=d \theta^{1} k_{1}+d \theta^{2} k_{2}
$$

( $k_{1}$ and $k_{2}$ are columns of bosonic superfields).

The integrability conditions (3.18) (or $\left(3.18^{\prime}\right)$ ) of the linear set (3.20) (or equivalently the Maurer Cartan equation for the graded group $S U(2 \mid 1))$ are:

$$
\begin{array}{r}
\left(D_{1}+a_{1}\right) k_{2}+\left(D_{2}+a_{2}\right) k_{1}-\left(A_{1} k_{2}+A_{2} k_{1}\right)=0 \\
D_{1} A_{2}+D_{2} A_{1}-\left\{A_{1}, A_{2}\right\}-k_{1} k_{2}^{\dagger}-k_{2} k_{1}^{\dagger}=0 .
\end{array}
$$

In the language of Eq. (4.1), the equations of motion of the $S U(2 \mid 1) / S\left(U_{2} \times U_{1}\right)$ $\sigma$-model read [17]:

$$
\left(D_{1}+a_{1}\right) k_{2}-\left(D_{2}+a_{2}\right) k_{1}-\left(A_{1} k_{2}-A_{2} k_{1}\right)=0 .
$$

Combining Eqs. (4.4a) and (4.5) one gets

$$
\begin{aligned}
& \left(D_{1}+a_{1}\right) k_{2}=A_{1} k_{2}, \\
& \left(D_{2}+a_{2}\right) k_{1}=A_{2} k_{1},
\end{aligned}
$$

and then

$$
D_{2}\left(k_{1}^{\dagger} k_{1}\right)=D_{1}\left(k_{2}^{\dagger} k_{2}\right)=0 .
$$

By exploiting local supersymmetry and conformal invariance of the $\sigma$-model, one 
can impose:

$$
k_{1}^{\dagger} k_{1}=k_{2}^{\dagger} k_{2}=m=\text { constant. }
$$

In this way we have performed the reduction of the $\sigma$-model and from now on we can follow the scheme outlined in ref. [11].

By using the gauge freedom

$$
\hat{\Omega} \rightarrow \hat{\Omega}^{\prime}=f^{-1} \hat{\Omega} f-f^{-1} \hat{D} f, \quad \forall f \in S\left(U_{2} \times U_{1}\right)
$$

of Eqs. (4.4) and (4.5), we can choose the following parametrization:

$$
k_{1}=i \sqrt{m}\left(\begin{array}{l}
e^{i H} \cos \Phi \\
e^{i(H+\alpha)} \sin \Phi
\end{array}\right), \quad k_{2}=\sqrt{m}\left(\begin{array}{l}
1 \\
0
\end{array}\right), \quad A_{1}=0,
$$

where $\Phi, \alpha$ and $H$ are 3 real superfields. In this gauge Eq. (4.4b) reads

$$
D_{1} A_{2}=m\left(\begin{array}{cc}
-2 \cos \Phi \sin H & -i e^{-i(H+\alpha)} \sin \Phi \\
i e^{i(H+\alpha)} \sin \Phi & 0
\end{array}\right) ;
$$

then, consistent with Eq. (4.11), one can choose $\left(A_{2}\right)_{22}=0$ and solve Eqs. (4.6) to express $A_{2}$ in terms of $\Phi, \alpha, H$ and of their derivatives. Inserting the result in Eq. (4.1), one gets:

$$
\Omega_{1}=i \sqrt{m}\left(\begin{array}{ccc}
0 & \lambda^{-1 / 2} e^{-i H} \cos \Phi & \lambda^{-1 / 2} e^{-i(H+\alpha)} \sin \Phi \\
\lambda^{-1 / 2} e^{i H} \cos \Phi & 0 & 0 \\
\lambda^{-1 / 2} e^{i(H+\alpha)} \sin \Phi & 0 & 0
\end{array}\right)
$$

$$
\Omega_{2}=\left(\begin{array}{ccc}
i \frac{\cos 2 \Phi}{\sin ^{2} \Phi} D_{2} H-i D_{2} \alpha & -\sqrt{\lambda m} & 0 \\
\sqrt{\lambda m} & i \frac{\cos 2 \Phi}{\sin ^{2} \Phi} D_{2} H-i D_{2} \alpha & e^{-i \alpha}\left(-D_{2} \Phi+i \cot \Phi D_{2} H\right) \\
0 & e^{i \alpha}\left(D_{2} \Phi+i \cot \Phi D_{2} H\right) & 0
\end{array}\right) .
$$

The arbitrary real constant $\lambda$ that appears in Eq. (4.12) is introduced by a Lorentz transformation ${ }^{5}$ :

$$
x^{+} \rightarrow \frac{x^{+}}{\lambda}, \quad x^{-} \rightarrow \lambda x^{-} ; \quad \theta^{1} \rightarrow \lambda^{-1 / 2} \theta^{1}, \quad \theta^{2} \rightarrow \sqrt{\lambda} \theta^{2} ;
$$

in such a way both Eqs. (4.4) and (4.5) can be interpreted as integrability conditions of the linear set (3.20), once that Lorentz invariance ( $\lambda$ independence) is required.

Substitution of (4.12) into the matrix equation (3.18) (or $\left(3.18^{\prime}\right)$ ) gives the following equations of motion:

$$
i D_{1} D_{2} \Phi-i \cot \Phi D_{1} \alpha D_{2} H+m \sin \Phi \cos H=0,
$$

5 We remark that the differentials $d \theta^{\alpha}$ have the same dimensions and the same transformation properties under the Lorentz group as the Grassmann variables $\theta^{\alpha}$ (while, of course, the opposite holds for the covariant derivatives $D_{\alpha}$ ) 


$$
\begin{aligned}
& i D_{1} D_{2} H-\frac{i}{\cos \Phi}\left(-\sin \Phi D_{1} \alpha D_{2} \Phi+\frac{1}{\sin \Phi} D_{1} \Phi D_{2} H\right)+m \frac{\sin ^{2} \Phi}{\cos \Phi} \sin H=0 \\
& i D_{1} D_{2} \alpha+\frac{i}{\sin \Phi \cos \Phi}\left(\cos 2 \Phi D_{1} \alpha D_{2} \Phi+\frac{1}{\sin ^{2} \Phi} D_{1} \Phi D_{2} H\right)-m \frac{\sin H}{\cos \Phi}=0
\end{aligned}
$$

Eqs. (4.13) can be simplified by noting that they imply the following equation:

$$
D_{2}\left(D_{1} H+\sin ^{2} \Phi D_{1} \alpha\right)=0
$$

whose simplest solution is

$$
D_{1} \alpha=-\frac{1}{\sin ^{2} \Phi} D_{1} H
$$

Eq. (4.15) allows us to get rid of $D_{1} \alpha$ from Eqs. (4.13) and to reproduce the equations of motion (2.4).

To eliminate the superfield $\alpha$ also from the matrix $\hat{\Omega}$, it is convenient to perform the gauge transformation (4.9) with

$$
f=\left(\begin{array}{ccc}
e^{-i \alpha} & 0 & 0 \\
0 & e^{-i \alpha} & 0 \\
0 & 0 & 1
\end{array}\right)
$$

By using Eq. (4.15) $\Omega_{1,2}$ become:

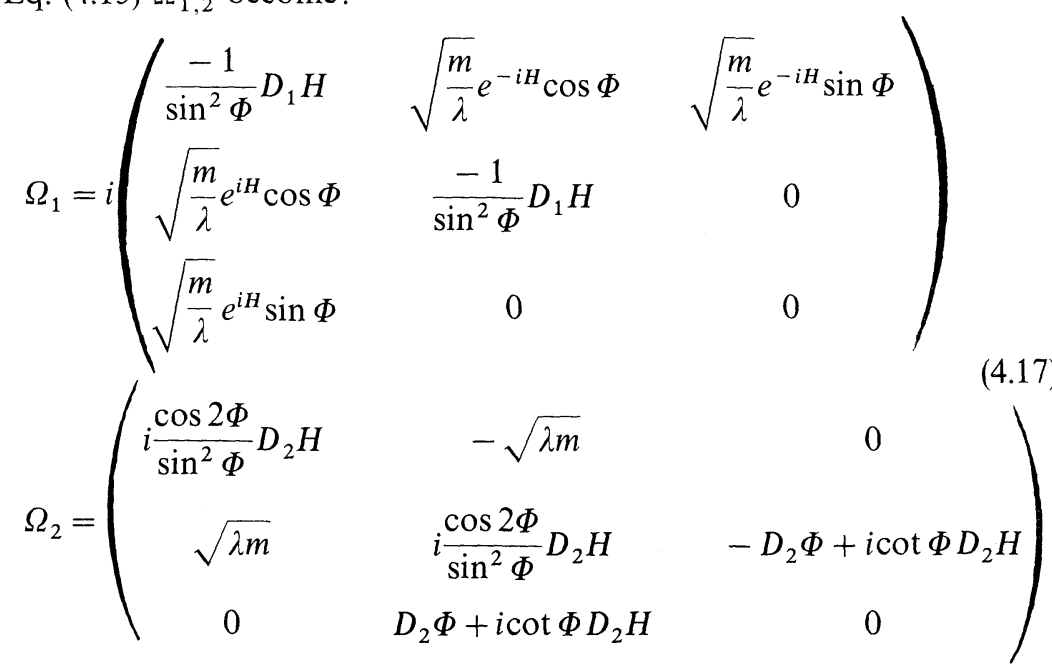

We have then succeeded in proving that the Lagrangian (2.1), with the potential (2.2), gives a set of equations of motion (2.4) which can also be interpreted as an integrability condition of the linear set (3.20), with $\hat{\Omega}$ given by Eqs. (3.14) and (4.17). In the next section we will use the "Lax set" (3.20) to find an infinite set of constants of motion of the model (2.1).

We wish to end this section recalling that the 1 -form $\omega$ (Eq. (3.13)) can be 
completed by calculating $\omega_{\mu}$ by Eqs. (3.19); the results are written in the Appendix. Here we only write the matrices $\omega_{\mu}$ of the linear set associated to the bosonic limit of the model; they are obtained by taking the $\theta$ independent part of $\omega_{\mu}$ (Eq. A.6) and sending to zero the fermionic fields $\psi$ and $\chi$. We get:

$$
\begin{aligned}
& \omega_{+}=\left(\begin{array}{ccc}
-i \frac{\partial_{+} h}{\sin ^{2} \varphi}+\frac{m}{i \lambda} & 0 & 0 \\
0 & -i \frac{\partial_{+} h}{\sin ^{2} \varphi}+\frac{m}{i \lambda} \cos ^{2} \varphi & \frac{m}{i \lambda} \sin \varphi \cos \varphi \\
0 & \frac{m}{i \lambda} \sin \varphi \cos \varphi & \frac{m}{i \lambda} \sin ^{2} \varphi
\end{array}\right) \\
& \omega_{-}=\left(\begin{array}{ccc}
i \frac{\cos 2 \varphi}{\sin ^{2} \varphi} \partial_{-} h+i \lambda m & 0 & 0 \\
0 & i \frac{\cos 2 \varphi}{\sin ^{2} \varphi} \partial_{-} h+i \lambda m & -\partial_{-} \varphi+i \cot \varphi \partial_{-} h \\
0 & \partial_{-} \varphi+i \cot \varphi \partial_{-} h & 0
\end{array}\right)
\end{aligned}
$$

with $\varphi$ and $h$ already introduced in the (2.3).

As expected, one easily checks that the consistency conditions (3.22) give back the GLRP equations (2.8). The most usual representation [7] of the linear set associate to the GLRP model

$$
\begin{aligned}
& \omega_{+}=-i\left(\begin{array}{lc}
\frac{\partial_{+} h}{2 \sin ^{2} \varphi}+\frac{m}{2 \lambda} \cos 2 \varphi & \frac{m}{2 \lambda} \sin 2 \varphi \\
\frac{m}{2 \lambda} \sin 2 \varphi & -\frac{\partial_{+} h}{2 \sin ^{2} \varphi}-\frac{m}{\lambda} \cos 2 \varphi
\end{array}\right) \\
& \omega_{-}=i\left(\begin{array}{lc}
\frac{\cos 2 \varphi}{2 \sin ^{2} \varphi} \partial_{-} h+\frac{\lambda m}{2} & i \partial_{-} \varphi+\cot \varphi \partial_{-} h \\
-i \partial_{-} \varphi+\cot \varphi \partial_{-} h & -\frac{\cos 2 \varphi}{2 \sin ^{2} \varphi} \partial_{-} h-\frac{\lambda m}{2}
\end{array}\right)
\end{aligned}
$$

is obtained by adding to $\omega_{\mu}$ (Eq. (4.18)) the diagonal matrices ${ }^{6} a_{\mu}$ with:

$$
\begin{aligned}
a_{+} & =\left(\frac{m i}{\lambda}+i \frac{\partial_{+} h}{\sin ^{2} \varphi}\right) C, \quad a_{-}=\left(-i m \lambda+i \frac{\cos 2 \varphi}{\sin ^{2} \varphi} \partial_{-} h\right) C ; \\
C & =\left(\begin{array}{ccc}
1 & \\
& \frac{1}{2} & \\
& & \frac{1}{2}
\end{array}\right),
\end{aligned}
$$

6 The equations of motion are not affected by this treatment as the diagonal matrices $a_{\mu}$ commute with the $\omega_{\mu}$ 's and the equation $\partial_{\mu} a_{v}-\partial_{v} a_{\mu}=0$ reproduces Eq. $(2.8 \mathrm{~b})$ 


\section{The Conservation Laws}

We wish to conclude this work by deriving an infinite set of conservation laws associated to the supersymmetric model (2.1). We follow the scheme of ref. [5]: having in mind the linear system

$$
\begin{aligned}
& D_{1} V=\Omega_{1} V \\
& D_{2} V=\Omega_{2} V
\end{aligned} \quad V=\left(\begin{array}{c}
V_{1} \\
V_{2} \\
V_{3}
\end{array}\right),
$$

with $\Omega_{\alpha}$ given by (4.17), we introduce the new bosonic superfields $U$ and $Y_{B}$ and the fermionic superfield $Y_{F}$ as follows:

$$
U=\ln V_{3}, \quad Y_{B}=\frac{V_{2}}{V_{3}}, \quad Y_{F}=\frac{V_{1}}{V_{3}} .
$$

Eqs. (5.1b) then become:

$$
\begin{aligned}
\sqrt{\lambda} Y_{B} & =-D_{2} Y_{F}-F Y_{F} Y_{B}+F^{\prime} Y_{F}, \\
\sqrt{\lambda} Y_{F} & =-F^{*}+D_{2} Y_{B}+F Y_{B} Y_{B}-F^{\prime} Y_{B}, \\
D_{2} U & =F Y_{B},
\end{aligned}
$$

where we have put $m=1$, and the fermionic quantities $F$ and $F^{\prime}$ are given by

$$
\begin{aligned}
F & =D_{2} \Phi+i \cot \Phi D_{2} H, \quad F^{*}=-D_{2} \Phi+i \cot \Phi D_{2} H, \\
F^{\prime} & =i \frac{\cos 2 \Phi}{\sin ^{2} \Phi} D_{2} H ;
\end{aligned}
$$

moreover the last of Eqs. (5.1a) takes the form:

$$
D_{1} U=\frac{i}{\sqrt{\lambda}} e^{i H} \sin \Phi Y_{F} .
$$

Eqs. (5.3c) and (5.5), whose consistency is guaranteed by the equations of motion (2.4), imply:

where

$$
D^{\alpha} J_{\alpha}=0,
$$

$$
J_{2}=-F Y_{B}, \quad J_{1}=\frac{i}{\sqrt{\lambda}} e^{i H} \sin \Phi Y_{F},
$$

and $Y_{B}(\lambda), Y_{F}(\lambda)$ are defined through the Riccati like equations $(5.3 \mathrm{a}, \mathrm{b})$.

Equation (5.6) is the supersymmetric way of writing a ( $\lambda$ depending) set of local conservation laws; in fact by expanding the spinorial superfield $J_{\alpha}(x, \theta)$ in powers of $\theta$ :

$$
J_{\alpha}(x, \theta)=\sigma_{\alpha}+i\left(\gamma^{\mu} \theta\right)_{\alpha} j_{\mu}+i\left[\left(A+\gamma_{5} B\right) \theta\right]_{\alpha}+\frac{i}{2} \theta^{\beta} \theta_{\beta} \rho_{\alpha}
$$

one can easily check that Eq. (5.6) implies

$$
\partial^{\mu} j_{\mu}(x)=0 .
$$


Eqs. (5.6), (5.7) can be put in a simpler form by eliminating $Y_{B}$ through Eq. (5.3a); one gets:

$$
J_{2}(\lambda)=\frac{F}{\sqrt{\lambda}}\left(D_{2} Y_{F}-F^{\prime} Y_{F}\right), J_{1}(\lambda)=\frac{i}{\sqrt{\lambda}} e^{i H} \sin \Phi Y_{F}
$$

and

$$
\sqrt{\lambda} Y_{F}=-F^{*}+\frac{1}{\sqrt{\lambda}}\left[-i \partial_{-} Y_{F}+\left(D_{2} F^{\prime}+F F^{*}\right) Y_{F}\right]+\frac{1}{\lambda}\left(D_{2} F-F F^{\prime}\right) Y_{F} \cdot D_{2} Y_{F}
$$

We can solve Eq. (5.9) in a recursive way by expanding $Y_{F}$ in power series of $\lambda^{-1}$

$$
Y_{F}=\frac{1}{i \sqrt{\lambda}} \sum_{n=0}^{\infty}(i \lambda)^{-n} Y^{(n+1 / 2)}
$$

by inserting Eq. (5.10) in Eq. (5.9) and equating the terms of the same power in $\lambda^{-1}$, one gets:

$$
\begin{gathered}
Y^{(1 / 2)}=-i F^{*}=i D_{2} \Phi+\cot \Phi D_{2} H, \\
Y^{(n+1 / 2)}=\left\{\partial_{-}+i\left(D_{2} F^{\prime}+F F^{*}\right)\right\} Y^{(n-1 / 2)}+i \sum_{l=1}^{n-1}\left(D_{2} F-F F^{\prime}\right) D_{2} Y^{(l-1 / 2)} \cdot Y^{(n-l-1 / 2)}, \\
n=1,2, \ldots
\end{gathered}
$$

Then Eqs. (5.6) and (5.7) become

$$
D^{\alpha} J_{\alpha}^{(n+1 / 2)}=0
$$

where the fermionic supersymmetric current $J_{\alpha}$ is

$$
\begin{aligned}
& J_{2}^{(n+1 / 2)}=F\left(D_{2} Y^{(n-1 / 2)}-F^{\prime} Y^{(n-1 / 2)}\right), \\
& J_{1}^{(n+1 / 2)}=i e^{i H} \sin \Phi Y^{(n-1 / 2)} .
\end{aligned}
$$

These expressions reproduce of course the results of the supersymmetric sineGordon model once $H$ is vanishing.

Before commenting on Eqs. (5.12) and (5.13) we explicitly remark that another set of conservation laws, which complete the bidimensional representations of the Lorentz group, can be obtained by choosing a different gauge in Eq. (4.10) $\left(A_{2}=0\right.$ instead of $A_{1}=0$ and $k_{1} \rightarrow k_{2}$ ).

It is interesting to observe as the (complex) conservation laws (5.12) work at least for the simplest case: the first conserved current $J_{\alpha}^{(3 / 2)}$ (that is the multiplet to which the energy momentum tensor belongs) reads:

$$
\begin{aligned}
J_{1}^{(3 / 2)}= & e^{i H}\left(-\sin \Phi D_{2} \Phi+i \cos \Phi D_{2} H\right) \equiv D_{2}\left(e^{i H} \cos \Phi\right) \\
J_{2}^{(3 / 2)}= & -\left[\partial_{-} \Phi D_{2} \Phi+\cot ^{2} \Phi \partial_{-} H D_{2} H\right. \\
& \left.+i \cot \Phi\left(\partial_{-} \Phi D_{2} H-\partial_{-} H D_{2} \Phi\right)\right] .
\end{aligned}
$$


The real and the imaginary part of Eq. (5.14) are:

$$
\begin{aligned}
i \operatorname{Im} J_{\alpha}^{(3 / 2)} & =\left(\begin{array}{l}
D_{2}(\cos \Phi \cos H) \\
-\partial_{-} \Phi D_{2} \Phi-\cot ^{2} \Phi \partial_{-} H D_{2} H
\end{array}\right) \stackrel{\text { def }}{=} I_{\alpha,-}, \\
-i \operatorname{Re} J_{\alpha}^{(3 / 2)} & =D_{2}\left(\begin{array}{l}
\cos \Phi \sin H \\
i \cot \Phi D_{2} \Phi D_{2} H
\end{array}\right) .
\end{aligned}
$$

The current $I_{\alpha,-}$ defined in Eq. (5.15a), together with its counterpart

$$
I_{\alpha,+}=\left(\begin{array}{c}
-\partial_{+} \Phi D_{1} \Phi-\cot ^{2} \Phi \partial_{+} H D_{1} H \\
D_{1}(\cos \Phi \cos H)
\end{array}\right)
$$

gives the supersymmetric generalization of the energy momentum tensor

$$
I_{\alpha \mu}=-\left[\partial \Phi \gamma_{\mu} D \Phi+\cot ^{2} \Phi \gamma_{\mu} D H-\gamma_{\mu} D(\cos \Phi \cos H)\right]_{\alpha}
$$

The supercurrent $I_{\alpha \mu}$ is related also to the generators of supersymmetry $Q_{\alpha}$; in fact it satisfies, beyond Eq. (5.12), the additional conservation

$$
\partial^{\mu} I_{\alpha \mu}=0
$$

then the $\theta$ independent part of $I_{\alpha \mu}$ is a conserved spinor current.

Of course the presence of this spinorial charge is not a surprise, as our model contains only superfields ( $\Phi$ and $H$ ) and their supersymmetric covariant derivatives; then its invariance under the supersymmetry transformations (3.6) is built in from the very beginning. However something unexpected can be learnt by looking to Eq. (5.15b); in fact the real part of the supersymmetrically conserved current $J_{\alpha}^{(3 / 2)}$ is a total covariant derivative. This suggests also that the bosonic supercurrent

$$
\begin{aligned}
& X_{1}=\cos \Phi \sin H, \\
& X_{2}=-i \cot \Phi D_{2} \Phi D_{2} H
\end{aligned}
$$

is conserved; this is indeed the case and the new conservation law

$$
D^{\alpha} X_{\alpha}=0
$$

holds.

The Lorentz multiplet can be completed by defining

$$
X_{\alpha \beta}=\left(\begin{array}{ll}
i \cot \Phi D_{1} \Phi D_{1} H & \cos \Phi \sin H \\
\cos \Phi \sin H & -i \cot \Phi D_{2} \Phi D_{2} H
\end{array}\right),
$$

which satisfies

$$
D^{\alpha} X_{\alpha \beta}=D^{\beta} X_{\alpha \beta}=0 .
$$

By expanding the bosonic supercurrent $X_{\alpha \beta}$ in powers of $\theta$ (analogously to Eq. (5.8)) one gets that Eq. (5.20') implies

$$
\partial^{\mu} \tau_{\mu \alpha}=0
$$

where $\tau_{\mu \alpha}(x)$ is a new conserved spinorial current.

Further comments and investigations on the new supersymmetry invariance 
related to this current are left to a forthcoming paper; here we wish only to note that the spinorial charge

$$
Q_{\alpha}^{\prime}=\int d x \tau_{\alpha}^{0}
$$

generates supersymmetry transformations which mix the component fields of $\Phi(x, \theta)$ and $H(x, \theta)$. Then the action (2.1) actually describes a model invariant under extended supersymmetry.

\section{Appendix}

In terms of component fields the Lagrangian (2.1) reads:

$$
\begin{aligned}
L= & -\frac{1}{2}\left\{\partial^{\mu} \varphi \partial_{\mu} \varphi+i \psi^{\alpha}(\partial \psi)_{\alpha}+\cot ^{2} \varphi\left[\partial^{\mu} h \partial_{\mu} h+i \chi^{\alpha}(\partial \chi)_{\alpha}\right]\right. \\
& \left.+\frac{1}{\sin ^{2} \varphi}\left[2 i \cot \varphi \psi^{\alpha}(\partial h \chi)_{\alpha}-\cot ^{2} \varphi \psi^{\alpha} \psi_{\alpha} \chi^{\beta} \chi_{\beta}\right]+m^{2} \sin ^{2} \varphi\right\} \\
& +i m \cos \varphi\left[-\frac{1}{2} \cos h\left(\psi^{\alpha} \psi_{\alpha}-\cot ^{2} \varphi \chi^{\alpha} \chi_{\alpha}\right)-\cot \varphi \sin h \psi^{\alpha} \chi_{\alpha}\right]
\end{aligned}
$$

where the auxiliary fields have already been eliminated. The corresponding equations of motion are:

$$
\begin{aligned}
& \square \varphi+\frac{\cos \varphi}{\sin ^{3} \varphi}\left[\partial^{\mu} h \partial_{\mu} h+2 i \cot \varphi \psi^{\alpha}(\widetilde{\partial} h \chi)_{\alpha}-\cot ^{2} \varphi \psi^{\alpha} \psi_{\alpha} \chi^{\beta} \chi_{\beta}\right] \\
& +i m \sin \varphi\left[\frac{1}{2} \cos h\left(\psi^{\alpha} \psi_{\alpha}-\cot ^{2} \varphi \chi^{\alpha} \chi_{\alpha}\right)+\cot \varphi \sin h \psi^{\alpha} \chi_{\alpha}\right] \\
& -m^{2} \sin \varphi \cos \varphi=0 \text {, } \\
& \square h-\frac{2}{\sin \varphi \cos \varphi}\left[\partial^{\mu} \varphi \partial^{\mu} h+i \cot \varphi \psi^{\alpha}(\widetilde{C} \varphi \chi)_{\alpha}\right] \\
& +i m \frac{1+\cos ^{2} \varphi}{\cos \varphi}\left[-\frac{1}{2} \sin h\left(\psi^{\alpha} \psi_{\alpha}-\cot ^{2} \varphi \chi^{\alpha} \chi_{\alpha}\right)+\cot \varphi \cos h \psi^{\alpha} \chi_{\alpha}\right]=0, \\
& \widetilde{\partial} \psi \cot \varphi\left({\frac{1}{\sin ^{2} \varphi}}^{\tau} h+m \cos \varphi \sin h\right) \cdot \chi \\
& +\cos \varphi\left(i \frac{\cot \varphi}{\sin ^{3} \varphi} \chi^{\alpha} \chi_{\alpha}+m \cos h\right) \cdot \psi=0 \\
& \partial \chi-\left(\frac{\partial \varphi}{\sin \varphi \cos \varphi}-\frac{i}{\sin ^{2} \varphi} \psi^{\alpha} \psi_{\alpha}+m \cos \varphi \cos h\right) \cdot \chi \\
& -\left(\frac{\dot{\partial} h}{\sin \varphi \cos \varphi}-m \sin \varphi \sin h\right) \cdot \psi=0 .
\end{aligned}
$$

One can directly check that they are the consistency conditions of the associate linear set:

$$
\partial_{\mu} V=\omega_{\mu}^{(0)} V
$$


where $\omega_{\mu}^{(0)}$ is the $\theta$ independent part of the superfield matrix $\omega_{\mu}$ given by Eq. (3.19); $\omega_{\mu}^{(0)}$ is a $3 \times 3$ antihermitian matrix whose elements are:

$$
\begin{aligned}
& \left(\omega_{+}^{(0)}\right)_{11}=\frac{1}{\sin ^{2} \varphi}\left(2 \cot \varphi \psi_{1} \chi_{1}-i \partial_{+} h\right)+\frac{m}{i \lambda},\left(\omega_{+}^{(0)}\right)_{12}=\sqrt{\frac{m}{\lambda}} e^{-i h} \sin \varphi\left(i \psi_{1}-\cot \varphi \chi_{1}\right) \\
& \left(\omega_{+}^{(0)}\right)_{13}=-\cot \varphi\left(\omega_{+}^{(0)}\right)_{12} \\
& \left(\omega_{+}^{(0)}\right)_{22}=\left(\omega_{+}^{(0)}\right)_{11}-\frac{m}{i \lambda} \sin ^{2} \varphi,\left(\omega_{+}^{(0)}\right)_{33}=\frac{m}{i \lambda} \sin ^{2} \varphi \\
& \left(\omega_{+}^{(0)}\right)_{23}=\frac{m}{i \lambda} \sin \varphi \cos \varphi \\
& \left(\omega_{-}^{(0)}\right)_{11}=\frac{1}{\sin ^{2} \varphi}\left(2 \cot \varphi \psi_{2} \chi_{2}+i \cos 2 \varphi \partial_{-} h\right)+i \lambda m,\left(\omega_{-}^{(0)}\right)_{12}=0 \\
& \left(\omega_{-}^{(0)}\right)_{13}=-i \sqrt{\lambda m}\left(i \psi_{2}+\cot \varphi \chi_{2}\right),\left(\omega_{-}^{(0)}\right)_{22}=\left(\omega_{-}^{(0)}\right)_{11}-2 \cot \varphi \psi_{2} \chi_{2} \\
& \left(\omega_{-0}^{(0)}\right)_{23}=-\partial_{-} \varphi+i \cot \varphi \partial_{-} h+2 \cot ^{2} \varphi \psi_{2} \chi_{2}, \\
& \left(\omega_{-}^{(0)}\right)_{33}=2 \cot \varphi \psi_{2} \chi_{2} .
\end{aligned}
$$

\section{References}

1. Di Vecchia, P., Ferrara, S.: Nucl. Phys. B130, 93-104 (1977); Witten, E.: Phys. Rev. D16, 2991-2994 (1977); see also Hruby, J.: Nucl. Phys. B131, 275-284 (1977)

2. Ferrara, S., Girardello, L., Sciuto, S.: Phys. Lett. 76B, 303-306(1978)

3. Marinucci, T., Sciuto, S.: Nucl. Phys. B156, 144-156(1979); Nissimov, E. R.: Nucl. Phys. B163, 374396 (1980)

4. Shankar, R., Witten, E.: Phys. Rev. D17, 2134-2143 (1978)

5. Girardello, L., Sciuto, S.: Phys. Lett. 77B, 267-269 (1978)

6. Chaichain, M., Kulish, P. P.: Phys, Lett. 78B, 413-416 (1978)

7. Pohlmeyer, K.: Commun. Math. Phys. 46, 707-718 (1976); Lund, F., Regge, T.: Phys. Rev. 14, 15241535 (1976)

8. Getmanov, B. S.: JETP Lett. 25, 119-122 (1977)

9. Getmanov, B. S.: Dubna Preprint E2-11093 (1977) (in Russian), and Dubna Preprint E2-80-354 (1980)

10. Sciuto, S.: Phys. Lett. 90B, 75-80 (1979)

11. D’Auria, R., Sciuto, S.: Nucl. Phys. B171, 189-208 (1980)

12. Zakharov, V. E., Mikhailov, A. V.: Sov. Phys. JETP 47, 1017-1027 (1978)

13. Berezin, F. A.: Sov. J. Nucl. Phys. 30, 605-609 (1979)

14. D’Auria, R., Regge, T., Sciuto, S.: Phys. Lett. 89B, 363-366 (1980), and Nucl. Phys. B171, 167-188 (1980)

15. Sohnius, M. F.: Nucl. Phys. B136, 461-474 (1978)

16. Lukierski, J.: Proceedings of IX-th Colloquium on Group Theoretic Methods, Cocoyoc (Mexico), June 1980. In: Lecture Notes in Physics, Vol. 135, p. 580. Berlin, Heidelberg, New York: Springer 1981

17. Napolitano, E., Sciuto, S.: Nuovo Cimento 64A, 406-420 (1981)

Communicated by R. Stora

Received July 20, 1981 